\title{
Selección de un método para la evaluación del impacto social usando AHP
}

\section{Selection of a method for social impact assessment using AHP}

\author{
Alexi Delgado ${ }^{1,2}$, I. Romero ${ }^{1}$ \\ ${ }^{1}$ Instituto Universitario de Investigación de Ingeniería del Agua y Medio Ambiente (IIAMA), Universitat \\ Politècnica de València, España. \\ 2 Universidad de Ciencias y Humanidades, Perú.
}

DOI: https://doi.org/10.33017/RevECIPeru2015.0013/

\section{Resumen}

El incremento de conflictos socio-ambientales durante la planificación o la ejecución de proyectos hacen que la evaluación del impacto social sea cada vez más necesaria y objetiva con el fin de prevenir posibles conflictos sociales. En este artículo realizamos un estudio para seleccionar la mejor alternativa metodológica disponible y aplicable a la evaluación del impacto social - social impact assessment (SIA) en proyectos y programas. La selección fue realizada aplicando la metodología del proceso analítico jerárquico - analytic hierarchy process (AHP). En este estudio se propusieron cuatro métodos alternativos para la evaluación del impacto social: Delphi, AHP, FAHP y agrupación Grey, los cuales fueron jerarquizados de acuerdo a los criterios de: Cuantificación, robustez y estandarización. Para realizar la selección usando AHP se contó con la participación de un panel de cuatro expertos seleccionados para este estudio. Los resultados mostraron que el mejor método para la evaluación del impacto social es la Evaluación grey complementado con el método Delphi y otros procedimientos cualitativos durante la fase de estudio de campo y recolección de información.

Descriptores: Evaluación del impacto social, Proceso analítico jerárquico (AHP)

\section{Abstract}

Increase of socio-environmental conflicts during the planning or execution of projects makes social impact assessment is increasingly necessary and objective in order to prevent potential social conflicts. In this paper, we present a study to select the best alternative methodology available and applicable to social impact assessment (SIA) on projects and programs. The selection was conducted using the methodology of analytic hierarchy process (AHP). In this study four alternative methods for social impact assessment were proposed: Delphi, AHP, FAHP and Grey clustering, which were ranked according to criteria: Quantification, robustness and standardization. To make the selection using AHP, we provided of a panel of four experts selected for this study. The results showed that the best method for social impact assessment is the Grey clustering complemented with Delphi method and other qualitative procedures during field study and data collection.

Keywords: Social Impact Assessment (SIA), Analytic Hierarchy Process (AHP)

\section{Introducción}

Los conflictos socio-ambientales se vienen incrementando debido al desarrollo de proyectos de inversión, que implican explotación de recursos naturales, que afectan a la población ubicada dentro de la zona de influencia de un proyecto determinado, como lo muestra, por ejemplo, el estudio realizado por Saqalli et al. [1] sobre conflictos socioambientales relacionados con la gestión del agua o el estudio realizado por Karjalainen y Järvikoski, acerca de conflictos socio-ambientales relacionados con explotación de recursos energéticos [2]. Para prevenir posibles conflictos socio-ambientales es 
necesario realizar la evaluación del impacto social de un proyecto antes, durante y después de su ejecución.

La evaluación del impacto social se ha realizado principalmente bajo enfoques cualitativos, como lo muestra, por ejemplo, el estudio desarrollado por Prenzel y Vanclay [3], quienes realizaron la evaluación del impacto social de un proyecto de desarrollo de una infraestructura. Los métodos cualitativos aportan mucha información durante el análisis de un problema, pero tienen limitaciones en el momento de la toma decisiones, porque no se dispone de información numérica que permita realizar una buena discriminación. Por lo tanto, estos métodos deben ser complementados con enfoques cuantitativos que permitan tener una evaluación más objetiva y faciliten la toma de decisiones.

Entre las alternativas metodológicas disponibles para cuantificar la información cualitativa durante la evaluación del impacto social (SIA) tenemos: Primero, el método Delphi usado, entre otras cosas, para realizar estudios de prospectiva y construir indicadores, como lo muestra el estudio hecho por García y Palomares [4], acerca de la construcción de indicadores para la evaluación de las instituciones universitarias. Segundo, el método AHP utilizado para la toma de decisiones, como lo muestra el estudio realizado por Sadeghi y Ameli [5]. Tercero, el método FAHP que es una extensión de AHP, que incorpora la lógica difusa en el proceso de tratamiento de datos. FAHP es un método que se usa, entre otras cosas, para la toma de decisiones como lo muestra el estudio realizado por Weck et al. [6], sobre la evaluación de alternativas para el ciclo de producción. Finalmente el método de agrupación Grey, el cual se utiliza para clasificar objetos usando criterios de evaluación, como lo muestra el estudio de Ke et al. [7], acerca de la evaluación del riesgo de inversión en un proyecto de energía.

El método AHP es una opción practica y útil para elegir la mejor, de entre un grupo de alternativas, como lo muestran los estudios realizados por Kim et al. [8] sobre la selección de alternativas de reciclaje, dentro de la gestión de residuos sólidos o el estudio realizado por Shenggang et al. [9] sobre el control de emergencias en accidentes de contaminación ambiental. Por lo tanto AHP es una herramienta que nos ayudará en la selección del mejor método para la SIA avaluando las cuatro alternativas metodológicas propuestas en este artículo.

Los criterios establecidos para la selección del mejor método para la SIA fueron: Cuantificación, robustez y estandarización. Estos criterios fueron seleccionados de acuerdo a los enfoques principales de calidad que debe tener un método en la opinión de Julia Vinagre [10] y mediante la consulta al equipo de expertos participante en este estudio.

Para realizar el proceso de selección de un método para la evaluación del impacto social (SIA) usando el proceso analítico jerárquico (AHP), se contó con la colaboración de un equipo de expertos formado por cuatro miembros, especialistas de las siguientes áreas: Métodos multicriterio, toma de decisiones, ciencias sociales y ciencias medioambientales. Las características de estos expertos es que además de conocer a profundidad su especialidad, también tienen una visión holística del problema socioambiental, requisito de integralidad de un experto en opinión de Landeta [11].

Los objetivos de este artículo son: Primero seleccionar el mejor método para la SIA usando AHP y segundo caracterizar el método finalmente elegido.

Este artículo está organizado de la siguiente forma: en la sección 2 se desarrolla la metodología, en la sección 3 se realiza la aplicación de AHP en la selección del método para la SIA, en la sección 4 se exponen los resultados y la discusión, y en la sección 5 son presentadas las conclusiones de este estudio.

\section{Metodología.}

La metodología usada en este artículo para la selección de un método para la SIA, es AHP. El método AHP fue propuesto en 1980 por Thomas L. Saaty [12]. AHP está diseñado para seleccionar la mejor de un conjunto de alternativas propuestas, evaluadas con respecto a ciertos criterios previamente establecidos [13]. El procedimiento de AHP se resume a continuación [14] y [15]:

Paso 1: Definir las alternativas para la evaluación: $A_{1}, A_{2}, A_{3}, . ., A_{m}$.

Paso 2: Definir los criterios para la evaluación: $C_{1}$, $C_{2}, C_{3}, \ldots, C_{n}$

Paso 3: Establecer la matriz de comparación y su consistencia.

Para determinar el peso de cada criterio se usa una matriz de comparación pareada, usando la escala propuesta por Saaty [16], cuyos valores se muestran en la Tabla 1. 
Tabla 1: Escala de Saaty de la importancia relativa.

\begin{tabular}{lcc}
\hline \multicolumn{1}{c}{ Escala } & $\begin{array}{c}\text { Calificación } \\
\text { numérica }\end{array}$ & Recíproco \\
\hline Muy recomendado & 9 & $1 / 9$ \\
De muy fuerte a extremadamente & 8 & $1 / 8$ \\
Muy fuertemente preferido & 7 & $1 / 7$ \\
De fuertemente a muy fuertemente & 6 & $1 / 6$ \\
Fuertemente preferido & 5 & $1 / 5$ \\
De moderadamente a fuertemente & 4 & $1 / 4$ \\
Moderadamente preferido & 3 & $1 / 3$ \\
De igualmente a moderadamente & 2 & $1 / 2$ \\
lgualmente preferido & 1 & 1 \\
\hline
\end{tabular}

Los resultados de la comparación entre los criterios: $C_{1}, C_{2}, C_{3}, \ldots, C_{n}$, quedan establecidos de acuerdo a la matriz mostrada en la Ecuación 1.

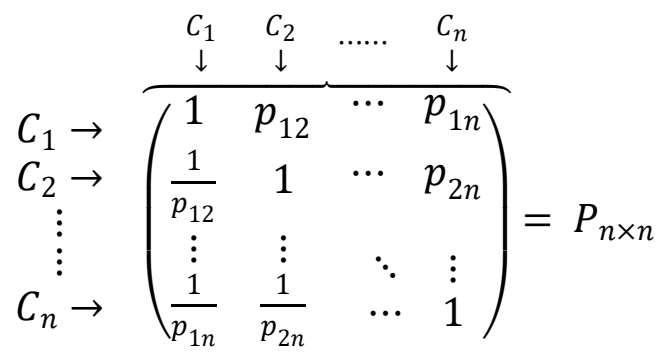

Ahora se normaliza la matriz $\mathrm{P}$ en cada columna dividiendo cada elemento entre la suma de la columna: $S_{1 \times n}=\left(\begin{array}{lllll}S_{1} & S_{2} & S_{3} & \cdots & S_{n}\end{array}\right)$. Luego se calcula el peso de cada criterio como la media aritmética de los elementos de cada fila en la matriz normalizada, obteniéndose la matriz de pesos de los criterios, como se indica en la Ecuación 2.

$$
W_{n \times 1}=\left(\begin{array}{lllll}
w_{1} & w_{2} & w_{2} & \cdots & w_{n}
\end{array}\right)^{t}
$$

Para determinar la consistencia de la matriz de comparación, primero calculamos el índice de consistencia - consistency index $(C l)$ de la matriz, usando la Ecuación 3.

$$
C I=\frac{\lambda_{\operatorname{Max}}-n}{n-1}
$$

Donde $n$ es el tamaño de la matriz y $\lambda_{\text {Max }}$ es el máximo autovector, el cual es calculando mediante la Ecuación 4.

$$
\lambda_{\text {Max }}=\sum_{i=1}^{n} s_{i} \cdot w_{i}
$$

Existen otros procedimientos para calcular $\lambda_{\text {Max }}$ y el peso de los criterios pero la diferencia de resultados es insignificante [16], por lo tanto el procedimiento desarrollado en este artículo es aceptado. Luego, se determina el índice de consistencia aleatoria random consistency index $(R I)$, cuyos valores dependen del tamaño de la matriz (n), los valores calculados por Saaty [13] se muestra en la Tabla 2.

Tabla 2: Valores de IR.

\begin{tabular}{ccccccccccc}
\hline $\boldsymbol{n}$ & $\mathbf{1}$ & $\mathbf{2}$ & $\mathbf{3}$ & $\mathbf{4}$ & $\mathbf{5}$ & $\mathbf{6}$ & $\mathbf{7}$ & $\mathbf{8}$ & $\mathbf{9}$ & $\mathbf{1 0}$ \\
\hline $\boldsymbol{I R}$ & 0 & 0 & 0,52 & 0,89 & 1,11 & 1,25 & 1,35 & 1,40 & 1,45 & 1,49 \\
\hline
\end{tabular}

Ahora, se calcula el ratio de consistencia consistency ratio $(C R)$ usando la Ecuación 5.

$$
C R=\frac{C I}{R I}
$$

La consistencia de la matriz es finalmente determinada comparando el ratio de consistencia $(C R)$ con los valores establecidos por Saaty [15] de acuerdo a la Tabla 3.

Tabla 3: Valores máximos del ratio de consistencia.

\begin{tabular}{cc}
\hline Tamaño de la matriz $(\boldsymbol{n})$ & Ratio de consistencia \\
\hline 3 & 0.05 \\
4 & 0.09 \\
5 o mayor & 0.10 \\
\hline
\end{tabular}

La matriz de comparación será consistente cuando el valor de $C R$ es menor que el valor indicado en la Tabla 3 de cuerdo al tamaño de la matriz.

Paso 4: Establecer el ranking de las alternativas para la decisión final.

Las alternativas: $A_{1}, A_{2}, A_{3}, . ., A_{m}$, son evaluadas de acuerdo a cada uno de los criterios: $C_{1}, C_{2}, C_{3}, \ldots$, $C_{n}$. Los resultados para cada criterio son presentados de acuerdo a la matriz mostrada en la Ecuación 6. 


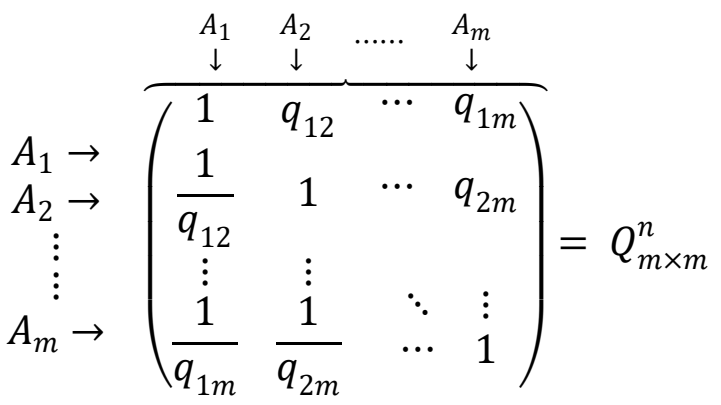

Luego de comprobar la consistencia de cada una de las matrices de comparación, se determina el peso de las alternativas con respecto a cada criterio obteniéndose la matriz mostrada en la Ecuación 7.

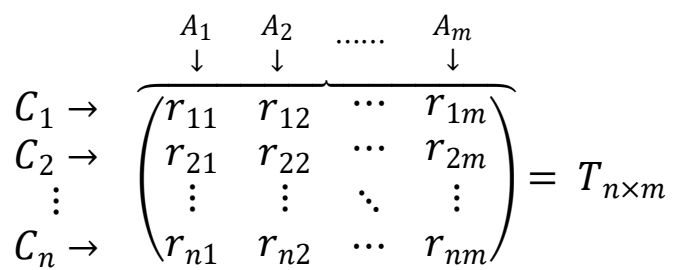

El ranking de las alternativas se establece de cuerdo a los resultados de la multiplicación de las matrices $W^{t}$ y T según la Ecuación 8.

$$
R_{1 \times m}=\left(W_{n \times 1}\right)^{t} \cdot T_{n \times m}
$$

\section{AHP en la selección del método para la SIA}

A continuación aplicamos AHP en la selección de un método para la SIA.

\subsection{Alternativas}

De acuerdo a la revisión de la literatura existente sobre: SIA, métodos para la evaluación del impacto ambiental, métodos de valoración multicriterio y la consulta al equipo de expertos; en este artículo se establecieron las siguientes alternativas:

\section{A1: Método Delphi}

El Método Delphi, según Landeta, se desarrolló desde mediados del siglo XX. "La denominación "Delphi" es la traducción inglesa de Delfos, ciudad de la antigua Grecia [...] celebre por los oráculos que Apolo realizaba por medio de una sacerdotisa (Pitia) [...] el método Delphi fue concebido en el seno del centro de investigación norteamericano The Rand Corporation a partir de finales de los años cuarenta [...] Un trabajo decisivo para el soporte científico de la técnica fue el llevado a cabo por Abraham Kaplan en 1949" [11]. Un Delphi consiste en la selección de un grupo de expertos a los que se les pregunta su opinión sobre cuestiones referidas a acontecimientos del futuro. Las estimaciones de los expertos se realizan en sucesivas rondas, anónimas, el objeto es tratar de conseguir consenso, pero con la máxima autonomía por parte de los participantes [17]. El método Delphi se ha aplicado a muchas investigaciones en las ciencias sociales, por ejemplo en el análisis de la prospectiva del sector audiovisual [18]. El método Delphi es una alternativa para la SIA porque permitiría mediante la consulta a expertos realizar la evaluación de un proyecto.

\section{A2: Método AHP}

Método AHP desarrollado en este artículo es una alternativa para la SIA, porque permitiría evaluar un proyecto en función de criterios sociales, los cuales estarían definidos de acuerdo a las particularidades del proyecto o programa en estudio.

\section{A3: Método FAHP}

El método FAHP incorpora a AHP los fundamentos de la lógica difusa (Fuzzy logic). Se considera como el inicio formal de la lógica difusa en 1965, cuando el profesor Lotfi A. Zadeh, publicó su trabajo "Fuzzy Sets" [19]. El método FAHP ha sido aplicado a una diversidad de problemas como por ejemplo para determinar la percepción de la calidad de servicios hoteleros [20] o para determinar los indicadores de la actividad emprendedora en una universidad [21] o para la evaluación de riesgos en la industria minera [22]. FAHP es una alternativa para SIA porque podría evaluar el impacto social usando criterios de forma similar a AHP pero basado en la lógica difusa.

\section{A4: Método de agrupación Grey}

El método de agrupación grey (Grey clustering) está basado en la teoría de sistemas grey desarrollado en 1985 por Deng [23], el cual estudia los problemas de muestras pequeñas y con información limitada, en el mundo real existen muchos problemas de este tipo, esto hace que su aplicación se extienda a muchos campos como por ejemplo la evaluación de páginas web [24] o la gestión del agua [25] o la gestión de la seguridad ocupacional [26]. El método de agrupación grey es una alternativa para SIA porque además de evaluar el impacto social usando criterios, establece intervalos o clases de grises para determinar un ranking del impacto social. 


\subsection{Criterios de selección}

Los criterios para la selección de un método para la SIA fueron establecidos en base a atributos que definen la calidad de un método [10] y la consulta al equipo de expertos que participó en el presente estudio, los criterios quedaron definidos como sigue:

\section{C1: Cuantificación}

Una de las limitaciones de la mayoría de los métodos para SIA hasta ahora existentes es la falta de capacidad para cuantificar los resultados, por lo tanto este criterio mide el nivel de cuantificación de los métodos analizados para la SIA.

\section{C2: Robustez}

Este criterio mide la solidez científica de los métodos para la SIA, analizando las teorías sobre las cuales se sustentan y el procedimiento que utilizan para sus evaluaciones.

\section{C3: Estandarización}

Este criterio mide el nivel de aplicabilidad de los métodos para SIA, es decir en qué medida el método puede ser aplicado a diferentes contextos y para la evaluación del impacto social de diferentes tipos de proyectos.

La jerarquía para la selección de un método para la SIA, incluyendo los criterios usados y las alternativas a ser evaluadas se muestra en la Figura 1.

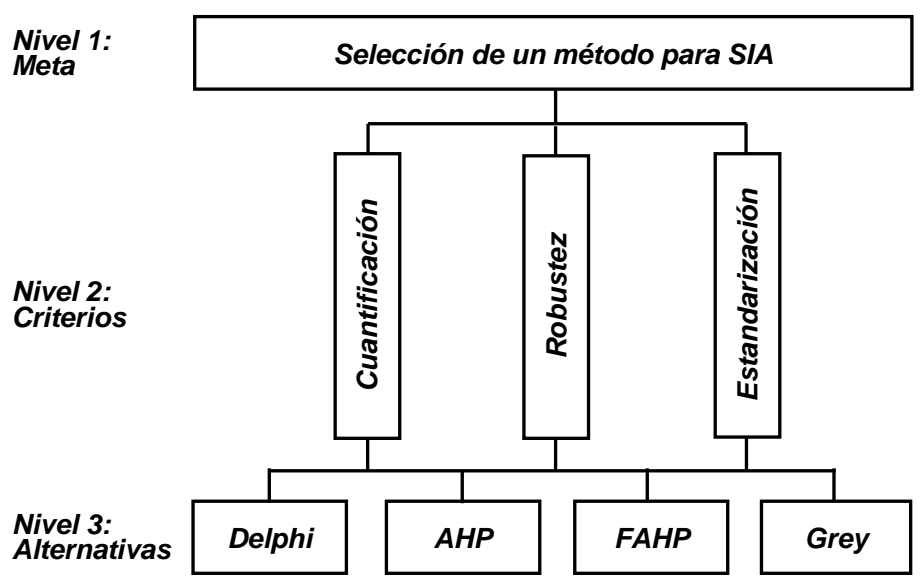

Figura 1: Jerarquía para la selección del método SIA

\subsection{Matrices e índice de consistencia}

El equipo de expertos: $E_{1}, E_{2}, E_{3}, E_{4}$, realizó la evaluación de los tres criterios, con el fin de establecer los pesos respectivos, de acuerdo a la escala de importancia relativa indicada en la Tabla 1.
Los resultados de la comparación pareada, proporcionada por los expertos son presentados en la Tabla 4.

Tabla 4: Comparación pareada entre los criterios.

\begin{tabular}{lccccc}
\hline & E1 & E2 & E3 & E4 & MG \\
\hline C1-C2 & 1.00 & 2.00 & 1.00 & 2.00 & 1.41 \\
C1-C3 & 2.00 & 3.00 & 0.50 & 3.00 & 1.73 \\
C2-C3 & 3.00 & 1.00 & 0.33 & 1.00 & 1.00 \\
\hline
\end{tabular}

Los resultados del equipo de expertos fueron agregados usando la media geométrica (MG) como lo propone Aznar [15]. La matriz de comparación acumulada se muestra en la Tabla 5.

Tabla 5: Matriz de comparación acumulada.

\begin{tabular}{cccc}
\hline & C1 & C2 & C3 \\
\hline$C 1$ & 1 & 1.41 & 1.73 \\
$C 2$ & 0.71 & 1 & 1.00 \\
$C 3$ & 0.58 & 1.00 & 1 \\
\hline$S$ & 2.28 & 3.41 & 3.73 \\
\hline
\end{tabular}

Luego, calculamos el peso de cada criterio y la consistencia de la matriz de comparación. Primero normalizamos la matriz dividendo cada elemento entre la suma de su respectiva columna, luego los pesos de cada criterio se calculan con el promedio aritmético de los elementos de cada fila. La matriz normalizada y los pesos de los criterios se muestran en la Tabla 6.

Tabla 6: Matriz de criterios normalizada.

\begin{tabular}{ccccc}
\hline & $c 1$ & $c 2$ & $c 3$ & $w$ \\
\hline$C 1$ & 0.44 & 0.41 & 0.46 & 0.44 \\
$C 2$ & 0.31 & 0.29 & 0.27 & 0.29 \\
$C 3$ & 0.25 & 0.29 & 0.27 & 0.27 \\
\hline
\end{tabular}

Para determinar la consistencia de la matriz primero calculamos el $\lambda_{\text {Max }}$ usando la Ecuación 4.

$\lambda_{\operatorname{Max}}=2.28 \times 0.44+3.41 \times 0.29+3.73 \times 0.27$

$\lambda_{\text {Max }}=3.0048$ 
Ahora, calculamos el índice de consistencia $(C l)$ para la matriz de tamaño $3 \times 3 \quad(n=3)$, usando la Ecuación 3.

$$
C I=\frac{3.0048-3}{3-1}=0.0024
$$

Según los valores de la Tabla 2 , para $n=3$, le corresponde un IR igual a 0.52, con lo cual se calcula el valor del ratio de consistencia $(C R)$ usando la Ecuación 5.

$$
C R=\frac{0.0024}{0.52}=0.0046
$$

En la Tabla 3 se observa que para $n=3$, el ratio de consistencia máximo es 0.05 y como el valor obtenido es $0.0046<0.05$, por lo tanto la matriz de comparación es consistente y los pesos obtenidos para los criterios son aceptables.

\subsection{Ranking de las alternativas}

Para establecer el ranking de las alternativas el equipo de expertos realizó la evaluación separada para cada criterio. Los resultados de las matrices de comparación agregadas de las alternativas con respecto a cada criterio se muestran en la Tabla 7.

Tabla 7: Matriz de comparación de las alternativas.

\begin{tabular}{lcccc}
\hline $\boldsymbol{C 1}$ & $\boldsymbol{A 1}$ & $\boldsymbol{A 2}$ & $\boldsymbol{A 3}$ & $\boldsymbol{A 4}$ \\
\hline $\boldsymbol{A 1}$ & 1 & 0.34 & 0.26 & 0.16 \\
$\boldsymbol{A} 2$ & 2.91 & 1 & 0.45 & 0.31 \\
$\boldsymbol{A 3}$ & 3.87 & 2.21 & 1 & 0.41 \\
$\boldsymbol{A 4}$ & 7.79 & 3.56 & 1.71 & 1 \\
$\boldsymbol{S}$ & 15.57 & 7.11 & 3.42 & 1.88 \\
\hline $\boldsymbol{C 2}$ & $\boldsymbol{A 1}$ & $\boldsymbol{A 2}$ & $\boldsymbol{A 3}$ & $\boldsymbol{A 4}$ \\
\hline $\boldsymbol{A 1}$ & 1 & 0.45 & 0.27 & 0.13 \\
$\boldsymbol{A 2}$ & 2.21 & 1 & 0.45 & 0.32 \\
$\boldsymbol{A 3}$ & 3.66 & 2.21 & 1 & 0.50 \\
$\boldsymbol{A 4}$ & 6.88 & 3.67 & 1.72 & 1 \\
$\boldsymbol{S}$ & 13.75 & 7.33 & 3.45 & 1.96 \\
\hline $\boldsymbol{C 3}$ & $\boldsymbol{A 1}$ & $\boldsymbol{A 2}$ & $\boldsymbol{A 3}$ & $\boldsymbol{A 4}$ \\
\hline $\boldsymbol{A 1}$ & 1 & 0.64 & 0.30 & 0.21 \\
$\boldsymbol{A 2}$ & 1.57 & 1 & 0.45 & 0.29 \\
$\boldsymbol{A 3}$ & 3.31 & 2.21 & 1 & 0.59 \\
$\boldsymbol{A 4}$ & 5.87 & 3.85 & 1.75 & 1 \\
$\boldsymbol{S}$ & 11.75 & 7.70 & 3.51 & 2.10 \\
\hline
\end{tabular}

Las matrices de comparación para las alternativas normalizadas y sus respectivos pesos se muestran en la Tabla 8.

Tabla 8: Matriz de alternativas normalizada.

\begin{tabular}{lccccc}
\hline $\boldsymbol{C 1}$ & $\boldsymbol{A 1}$ & $\boldsymbol{A 2}$ & $\boldsymbol{A 3}$ & $\boldsymbol{A 4}$ & $\boldsymbol{T 1}$ \\
\hline $\boldsymbol{A 1}$ & 0.06 & 0.05 & 0.08 & 0.09 & 0.07 \\
$\boldsymbol{A 2}$ & 0.19 & 0.14 & 0.13 & 0.17 & 0.16 \\
$\boldsymbol{A 3}$ & 0.25 & 0.31 & 0.29 & 0.22 & 0.27 \\
$\boldsymbol{A 4}$ & 0.50 & 0.50 & 0.50 & 0.53 & 0.51 \\
\hline $\boldsymbol{C 2}$ & $\boldsymbol{A 1}$ & $\boldsymbol{A 2}$ & $\boldsymbol{A 3}$ & $\boldsymbol{A 4}$ & $\boldsymbol{T 2}$ \\
\hline $\boldsymbol{A 1}$ & 0.07 & 0.06 & 0.08 & 0.07 & 0.07 \\
$\boldsymbol{A 2}$ & 0.16 & 0.14 & 0.13 & 0.17 & 0.15 \\
$\boldsymbol{A 3}$ & 0.27 & 0.30 & 0.29 & 0.26 & 0.28 \\
$\boldsymbol{A 4}$ & 0.50 & 0.50 & 0.50 & 0.51 & 0.50 \\
\hline $\boldsymbol{C 3}$ & $\boldsymbol{A 1}$ & $\boldsymbol{A 2}$ & $\boldsymbol{A 3}$ & $\boldsymbol{A 4}$ & $\boldsymbol{T 3}$ \\
\hline $\boldsymbol{A 1}$ & 0.09 & 0.08 & 0.09 & 0.10 & 0.09 \\
$\boldsymbol{A 2}$ & 0.13 & 0.13 & 0.13 & 0.14 & 0.13 \\
$\boldsymbol{A 3}$ & 0.28 & 0.29 & 0.29 & 0.28 & 0.28 \\
$\boldsymbol{A 4}$ & 0.50 & 0.50 & 0.50 & 0.48 & 0.49 \\
\hline
\end{tabular}

La Tabla 3, nos indica que para una matriz de $4 \times 4$ $(n=4)$ el ratio de consistencia máximo es 0.09 y los valores calculados para las matrices de comparación de alternativas para cada criterio son: 0.0166, 0.0001 y 0.0373 , respectivamente, vemos que los tres valores son menores a 0.09 , por lo tanto las tres matrices son consistentes y los pesos de las alternativas en cada criterio son válidas.

Finalmente usando la Ecuación 8, calculamos el ranking de las alternativas. La matriz de pesos de los criterios es:

$$
W_{3 \times 1}=\left(\begin{array}{lll}
0.44 & 0.29 & 0.27
\end{array}\right)^{t}
$$

La matriz de pesos de las alternativas con respecto a cada criterio es:

$$
T_{3 \times 4}=\left(\begin{array}{llll}
0.07 & 0.16 & 0.27 & 0.51 \\
0.07 & 0.15 & 0.28 & 0.50 \\
0.09 & 0.13 & 0.28 & 0.49
\end{array}\right)
$$

Ahora calculamos el ranking de las alternativas:

$$
R_{1 \times 4}=\left(W_{3 \times 1}\right)^{t} \cdot T_{3 \times 4}
$$

$R_{1 \times 4}=\left(\begin{array}{lll}0.44 & 0.29 & 0.27\end{array}\right)\left(\begin{array}{llll}0.07 & 0.16 & 0.27 & 0.51 \\ 0.07 & 0.15 & 0.28 & 0.50 \\ 0.09 & 0.13 & 0.28 & 0.49\end{array}\right)$ 


$$
\left.R_{1 \times 4}=\begin{array}{cccc}
0.07 & 0.15 & 0.28 & 0.50
\end{array}\right)
$$

\section{Resultados y discusión}

En la Figura 2, se muestran los resultados de la aplicación del AHP, en la cual se observa que el mejor método para la SIA es el método de agrupación Grey, en segundo lugar se ubica el método FAHP, en tercer lugar está el método AHP y en el cuarto lugar está el método Delphi.

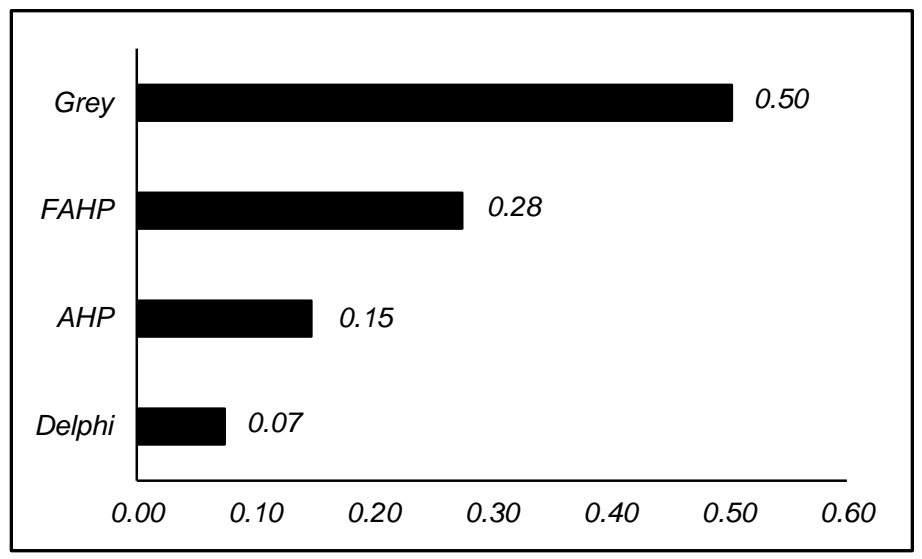

Figura 2: Ranking de las alternativas para SIA

A continuación analizamos las potencialidades de cada uno de los métodos para la SIA, recogidas desde la revisión de la literatura y la opinión del equipo de expertos.

Las ventajas del método Delphi son: Los expertos pueden argumentar sus respuestas de manera anónima, la opinión de un equipo de expertos podría considerarse como subjetiva pero no es arbitraria, por los tanto los resultados son fiables y Delphi puede ser considerado como un complemento para otras metodologías para la SIA. Las desventajas del método Delphi son: El uso de una estadística descriptiva en el tratamiento de los datos disminuyen su capacidad de cuantificación, además si se realizan muchas rondas, los expertos tienden a cambiar sus opiniones.

El método AHP tiene las siguientes ventajas: El uso de criterios de evaluación y la determinación de pesos para cada criterio eleva el nivel de objetividad de la evaluación con respecto a Delphi, por otro lado los cálculos relativamente sencillos hacen que este método se aplique a diferentes casos de estudio. La desventaja de AHP es su base matemática relativamente sencilla que requiere ser complementada con otras teorías para lograr una SIA más objetiva.
La ventaja del método FAHP con respecto a AHP es que eleva el nivel de cuantificación debido a que usa la teoría de la lógica difusa en el proceso de cálculo. Es importante tener cuidado en la recolección de los datos desde los expertos, para tener resultados óptimos al aplicar el método FAHP. En muchos casos no se dispone de datos cuantitativos para facilitar la valoración de los expertos, eso hace que la valoración sea subjetiva, por eso es necesario previamente realizar una cuantificación de las variables haciendo uso de la teoría de sistemas grey.

El método de agrupación Grey tiene ventaja respecto al método FAHP porque además de tener un buen nivel de cuantificación por la aplicación de los sistemas grey en el tratamiento de los datos, incorpora el uso de las clases de grises en cada criterio para realizar la SIA, lo cual permite tener un mayor rango de evaluación para los expertos y por lo tanto facilita el recojo de la información.

Finalmente de acuerdo a la opinión del equipo de expertos y el análisis de la literatura existente, el mejor método para realizar la evaluación del impacto social de proyectos y programas es el método de agrupación Grey, que puede ser complementado con un Delphi en la etapa de recojo de información. Por otro lado, para realizar la evaluación del impacto social es necesario analizar los grupos de interés existentes dentro de la población afectada, ya que cada uno de ellos puede tener una valoración del impacto social diferente, por lo tanto es necesario realizar la evaluación del impacto social del proyecto en cada grupo de interés y luego establecer comparaciones entre ellos para prevenir posibles conflictos socio-ambientales.

\section{Conclusiones}

El método AHP nos permitió seleccionar el mejor método para la SIA mediante la evaluación de un equipo de expertos que finalmente estableció que el mejor método es la agrupación Grey debido a la facilidad al momento del recojo de información desde la población afectada y el buen nivel de objetividad y cuantificación por la teoría matemática en la que está basada.

El método de agrupación Grey para la SIA debería ser complementado con un Delphi en el momento del recojo de información desde la población afectada y el equipo de expertos que evalúa un determinado proyecto, además es conveniente realizar una evaluación del impacto social en cada grupo de interés dentro de la población afectada con el fin de determinar sus diferencias y proponer medidas para prevenir posibles conflictos socioambientales. 
En futuras investigaciones, el método de agrupación Grey se puede aplicar en la evaluación del impacto social de proyectos tales como: Proyectos hídricos, proyectos de explotación minera, proyectos de explotación de hidrocarburos, proyectos de construcción de obras públicas, etc. Este método también podría ser aplicado para la evaluación del impacto social de programas públicos o privados.

\section{Agradecimientos}

Queremos agradecer al equipo anónimo de expertos que con su colaboración y sugerencia hicieron posible la realización de este estudio.

\section{Referencias}

[1] M. Saqalli, S. Thiriot, F. Amblard. Investigating Social Conflicts Linked to Water Resources Through Agent-Based Modelling. NATO Science for Peace and Security Series. 75 (2010) 142-157.

[2] T.P. Karjalainen, T. Järvikoski. Negotiating River Ecosystems: Impact Assessment and Conflict Mediation in the Cases of Hydro-Power Construction. Environmental Impact Assessment Review. 30(5) (2010) 319-327.

[3] P. Prenzel, F. Vanclay. How Social Impact Assessment can Contribute to Conflict Management. Environmental Impact Assessment Review. 45 (2014) 30-37.

[4] A. García, M. Palomares. Indicadores Para La Evaluación De Las Instituciones Universitarias: Validación a Través Del Método Delphi. Universitat Politècnica de València. ed.CSIC, 2012.

[5] M. Sadeghi, A. Ameli. An AHP Decision Making Model for Optimal Allocation of Energy Subsidy among Socio-Economic Subsectors in Iran. Energy Policy. 45 (2012) 24-32.

[6] M. Weck, F. Klocke, H. Schell, E. Rüenauver. Evaluating Alternative Production Cycles using the Extended Fuzzy AHP Method. European Journal of Operational Research. 100 (1997) 351-366.

[7] L. Ke, S. Xiaoliu, T. Zhongfu, G. Wenyan. Grey Clustering Analysis Method for Overseas Energy Project Investment Risk Decision. Systems Engineering Procedia. 3(2012) 55-62.

[8] M. Kim, Y. Jang, S. Lee. Application of Delphi- AHP Methods to Select the Priorities of WEEE for Recycling in a Waste Management DecisionMaking Tool. Journal of Environmental Management. 128 (2013) 941-948.

[9] S. Shenggang, C. Jingcan, F. Li, L. Wenyan, Z. Liqiu. Construction of a Technique Plan Repository and Evaluation System based on AHP Group Decision-Making for Emergency Treatment And disposal in Chemical Pollution Accidents. Journal of Hazardous Materials. 276(2014) 200-206.
[10] Julia Vinagre. Calidad De Métodos Analíticos. Santiago de Chile, ed. FAO, 1995.

[11] J. Landeta. El Método Delphi, Una Técnica De Previsión Del Futuro. Barcelona: Editorial Ariel, 2002.

[12] T. Saaty. The Analytic Hierarchy Process. New York: McGraw-Hill, 1980.

[13] T. Saaty, L. Vargas. Models, Methods, Concepts \& Applications of the Analytic Hierarchy Process. $2^{\circ}$ ed. New York: Springer, 2012.

[14] F. Ahammeda, A. Azeem. Selection of the most Appropriate Package of Solar Home System using Analytic Hierarchy Process Model in Rural Areas of Bangladesh. Renewable Energy. 55(2013) 6-11.

[15] J. Aznar, F. Guijarro. Nuevos Métodos De Valoración, Modelos Multicriterio. $2^{\mathrm{a}}$ ed. Valencia: Universitat Politèctica de València, 2012.

[16] R. Vargas. Utilizando El Proceso Analítico Jerárquico Para Seleccionar Y Priorizar Proyectos En Una Cartera. Washington DC 2010.

[17] E. Astigarraga. El Método Delphi. España: Universidad de Deusto-San Sebastian, 2005.

[18] F. Ortega. El Método Delphi, Prospectiva En Las Ciencias Sociales. Revista EAN. 64(2008) 31-54.

[19] L. Zadeh. Fuzzy Sets. Information Control. 8(1965) 338-353.

[20] Y. Chen, T. Yu, P. Tsui, C. Lee. A Fuzzy AHP Approach to Construct International Hotel Spa Atmosphere Evaluation Model. Qual Quant. 48(2014) 645-657.

[21] K. Reza. Indicators of Entrepreneurial University: Fuzzy AHP and Fuzzy TOPSIS Approach. J Knowl Econ. 5(2014) 370-387.

[22] S. Verma, S. Chaudhri. Integration of Fuzzy Reasoning Approach (FRA) and Fuzzy Analytic Hierarchy Process (FAHP) for Risk Assessment in Mining Industry. Journal of Industrial Engineering and Management. 7(2014) 1347-1367.

[23] J. Deng. Generation Functions of Grey Systems. Fuzzy Mathematics. 5(1985) 11-22.

[24] Ch. Bindu, M. Padmaja, J. Chandulal. Evaluating Web Sites Based on GHAP. International Journal on Computer Science and Engineering. 2(2010) 674-679.

[25] L. Zhang, F. Wu, P. Jia. Grey Evaluation Model Based on Reformative Triangular Whitenization Weight Function and its Application in Water Rights Allocation System. The Open Cybernetics \& Systemics Journal. 7(2013) 1-10.

[26] C. Li, K. Chen, X. Xiang. An Integrated Framework for Effective Safety Management Evaluation: Application of an Improved Grey Clustering Measurement. Expert Systems with Applications. 42(2015) 5541-5553.

Email:

kidelvil@doctor.upv.es

alexidelgado@yahoo.es 